\title{
Expression of adiponectin receptors, AdipoR1 and AdipoR2, in normal colon epithelium and colon cancer tissue
}

\author{
KYOKO YONEDA $^{1}$, AYAKO TOMIMOTO ${ }^{1}$, HIROKI ENDO ${ }^{1}$, HIROSHI IIDA ${ }^{1}$, MICHIKO SUGIYAMA ${ }^{1}$, \\ HIROKAZU TAKAHASHI ${ }^{1}$, HIRONORI MAWATARI ${ }^{1}$, YUICHI NOZAKI ${ }^{1}$, KOJI FUJITA ${ }^{1}$, MASATO YONEDA ${ }^{1}$, \\ MASAHIKO INAMORI ${ }^{1}$, NORIKO NAKAJIMA ${ }^{2}$, KOICHIRO WADA ${ }^{3}$, YOJI NAGASHIMA ${ }^{4}$, \\ HITOSHI NAKAGAMA ${ }^{5}$, HIROSHI UOZAKI ${ }^{6}$, MASASHI FUKAYAMA ${ }^{6}$ and ATSUSHI NAKAJIMA ${ }^{1}$ \\ ${ }^{1}$ Gastroenterology Division, Yokohama City University School of Medicine, 3-9 Fuku-ura, Kanazawa-ku, Yokohama; \\ ${ }^{2}$ Department of Pathology, National Institute of Infectious Diseases, 1-23-1 Toyama, Shinjuku-ku, Tokyo; \\ ${ }^{3}$ Department of Pharmacology, Graduate School of Dentistry, Osaka University, 1-8 Yamadaoka, Suita, Osaka; \\ ${ }^{4}$ Department of Molecular Pathology, Yokohama City University School of Medicine, 3-9 Fuku-ura, Kanazawa-ku, \\ Yokohama; ${ }^{5}$ Biochemistry Division, National Cancer Center Research Institute, 1-1 Tsukiji 5-chome, Chuo-ku, Tokyo; \\ ${ }^{6}$ Department of Pathology, Graduate School of Medicine, The University of Tokyo, 7-3-1 Hongo, Bunkyo-ku, Tokyo, Japan
}

Received March 20, 2008; Accepted May 2, 2008

DOI: 10.3892/or_00000031

\begin{abstract}
Adiponectin is secreted by adipocytes and is a key hormone responsible for insulin sensitization. Recent studies have shown that plasma adiponectin is decreased in patients with breast, endometrial and gastric cancer. However, the effect of adiponectin on colorectal carcinogenesis is controversial. It is now well known that the adiponectin receptor exists in two isoforms, adiponectin receptor 1 (AdipoR1) and adiponectin receptor 2 (AdipoR2). We examined the expression of the adiponectin receptors on normal colon mucosa and colon cancer tissues in a human study using real-time RT-PCR, Western blotting and immunohistochemical staining. Adiponectin receptors, AdipoR1/ AdipoR2, were expressed in normal colon epithelial and colon cancer cells. Furthermore, laser microdissection was performed to confirm our results. These results suggest that adiponectin may exert some effects on normal colon epithelium or colon cancer cells directly through adiponectin receptors. Further studies are required to elucidate the function of the AdipoRs activated by adiponectin and the downstream mechanisms of AdipoRs in colon cancer cells.
\end{abstract}

\section{Introduction}

Adipose tissue secretes several bioactive substances known as adipocytokines and obesity is an important risk factor for

Correspondence to: Dr Atsushi Nakajima, Gastroenterology Division, Yokohama City University School of Medicine, 3-9 Fuku-ura, Kanazawa-ku, Yokohama, Japan

E-mail: nakajima-tky@umin.ac.jp

Key words: colon cancer, adiponectin, adiponectin receptor many diseases, including diabetes mellitus (DM) $(1,2)$. Human adiponectin, earlier known as ACRP30 or AdipoQ, is a $30 \mathrm{kDa}$ adipokine composed of 247 amino acids, which is encoded by the apMl gene and is located on chromosome 3, locus 3q27 (3-5). Adiponectin is secreted by adipocytes and is a key hormone responsible for insulin sensitization. While the adiponectin protein is abundantly found in the plasma of healthy human subjects, its plasma levels are dramatically decreased in patients with obesity and/or type 2 DM $(2,3,6)$. Since obesity and type $2 \mathrm{DM}$ have been reported to be associated with an elevated risk of colorectal cancer (CRC) (7), it has been speculated that the plasma level of adiponectin may be related to the risk of CRC. Furthermore, previous studies have shown that decreased levels of plasma adiponectin were observed in patients with breast, endometrial and gastric cancer (8-11). However, several contradictory results have been reported from human clinical studies on the relationship between the plasma levels of adiponectin and the risk of CRC $(12,13)$. It is now well known that the adiponectin receptor exists in two isoforms; adiponectin receptor 1 (AdipoR1), which is abundantly expressed in the skeletal muscle and adiponectin receptor 2 (AdipoR2), which is predominantly expressed in the liver (14). Recent studies have shown that the adiponectin receptors are expressed in human breast (15) and prostate cancer (16). Takahata et al showed that AdipoR1 and AdipoR2 (AdipoRs) were expressed in normal breast epithelial cells and breast cancer cells (15). However, it remains unclear whether the adiponectin receptors are also expressed in the normal colon mucosa and colon cancer tissue. In this study, we examined at genetic and protein expression levels the expression of AdipoRs on normal colon mucosa and colon cancer tissues from patients.

\section{Materials and methods}

Patients. We evaluated patients with CRC and normal subjects who underwent colonoscopy at Yokohama City University 
A
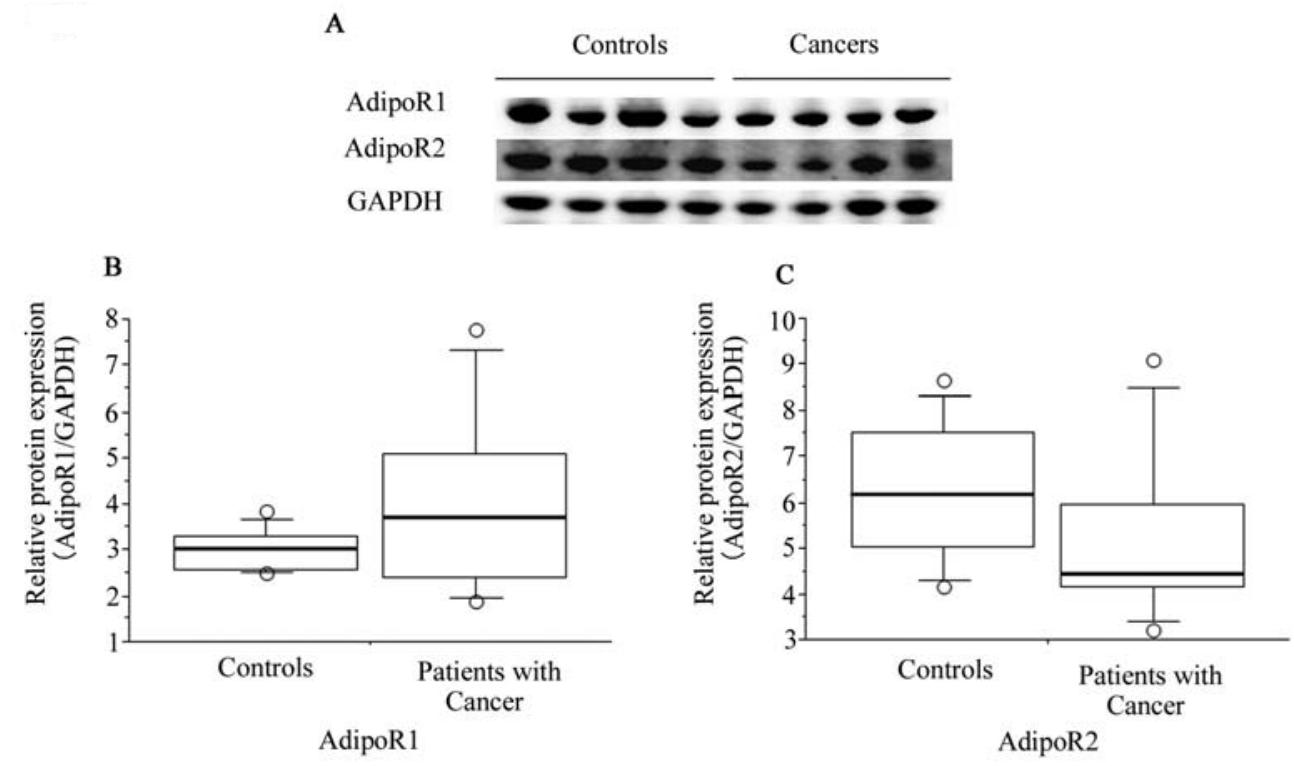

Figure 1. (A) The protein expression levels of AdipoR1 and AdipoR2 were examined with Western blotting. (B and C) Box plots showing interquartile range (box), median (thick line), range (thin line) and outliers (circles). Values are normalized to the level of GAPDH as the internal control. AdipoR1 (B) and AdipoR2 (C) in patients with cancer and control subjects. All samples expressed the AdipoR1 and AdipoR2 proteins, with no significant difference seen in these levels between the control and cancer specimens.

Hospital from April to September 2007 after informed consent was provided. A total of 34 cases were studied. CRC was diagnosed by histological analysis. The study was conducted with the approval of the Ethics Committee of Yokohama City University.

Western blotting. Biopsy specimens were homogenized and resolved by polyacrylamide gel (Bio-Rad Japan, Tokyo, Japan). Proteins were transferred onto a Hybond-P PVDF transfer membrane (Amersham Biosciences, Little Chalfont, UK) and incubated with antibodies to AdipoR1 (1:2000) or AdipoR2 (1:1000, Santa Cruz Biotechnology, Santa Cruz, CA, USA) as primary antibodies. For the internal control, membranes were incubated with an antibody to GAPDH (1:1000, Trevigen, Gaithersburg, MD, USA) as the primary antibody. Immune complexes were detected with the ECL Western blot system (Amersham Biosciences).

Real-time reverse transcriptase-polymerase chain reaction (real-time $R T-P C R$ ). Total RNA from the biopsy specimens was purified using an RNeasy kit (Qiagen, Hilden, Germany). Real-time RT-PCR was carried out using Taq Man reverse transcription reagents and Taq Man gene expression master mix (Applied Biosystems). The primers were AdipoR1, AdipoR2 and $B$-actin (Applied Biosystems). The expression levels of AdipoRI and AdipoR2 were shown as a ratio to $B$-actin as the internal control. To compare the results achieved in colon RNA with liver and skeletal muscle mRNA, human liver total RNA and human skeletal muscle total RNA (Clontech Laboratories, Mountain View, CA, USA) were also evaluated using real-time PT-PCR.

Laser microdissection and real-time RT-PCR. Frozen biopsy specimens were cut into $7-\mu \mathrm{m}$ sections and stained with toluidine blue (Muto Pure Chemicals, Tokyo, Japan). The cancer cells and normal cells were collected with PALM
MicroLaser Systems (P.A.L.M. Microlaser Technologies, Bernried, Germany). Total RNA was purified using an RNAqueous-micro kit (Applied Biosystems). The real-time RT-PCR procedure was performed as described above.

Immunohistochemical staining. Formalin-fixed and paraffinembedded samples were deparaffinized and rehydrated. The sections were incubated with antibodies to AdipoR1 (1:400) and AdipoR2 (1:400, Phoenix Parmaceuticals) as the primary antibodies using an LSAB2 kit (Dako cytomation). The sections were incubated with biotinylated immunoglobulin as the secondary antigen and were then treated with peroxidaseconjugated streptavidin. The antibody complex was visualized with 3,3'-diaminobenzidine, tetrahydrochloride (Dojindo Laboratories, Kumamoto, Japan).

Statistical analysis. The statistical analyses were performed with StatView-J 5.0 (SAS Institute, Cary, NJ, USA). The association between the clinicopathological factors and expression levels of the AdipoRs was assessed with the Student's t-test. P-values of $<0.05$ were considered significant.

\section{Results}

Patients. The characteristics of the CRC patients and control subjects are shown in Table I. The CRC patients $(n=16)$ and control subjects $(n=18)$ were similar with regard to age and body mass index (BMI). Patients using drugs which could influence serum adiponectin levels and patients with inflammatory bowel diseases were excluded from the present study.

Western blotting of AdipoR1 and AdipoR2. We examined the protein expression levels of AdipoRs using Western blotting (Fig. 1). The expression levels of the AdipoRs were detected in the controls and colon cancer tissues (Fig. 1A). All samples 

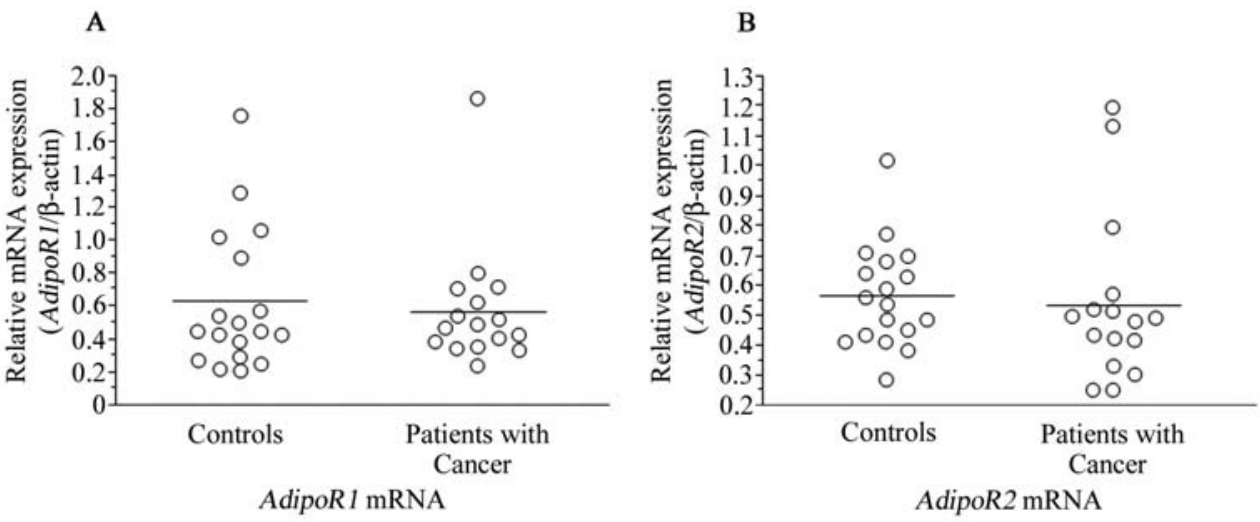

Figure 2. Colonic mRNA expression levels of AdipoR1 (A) and AdipoR2 (B) in patients with cancer and control subjects were evaluated using real-time RT-PCR Values are normalized to the level of $B$-actin. Bar, mean value of patients with cancer and control subjects. All samples expressed AdipoRl and AdipoR2 mRNA, the expression levels of which were not significantly different between the control and cancer specimens.
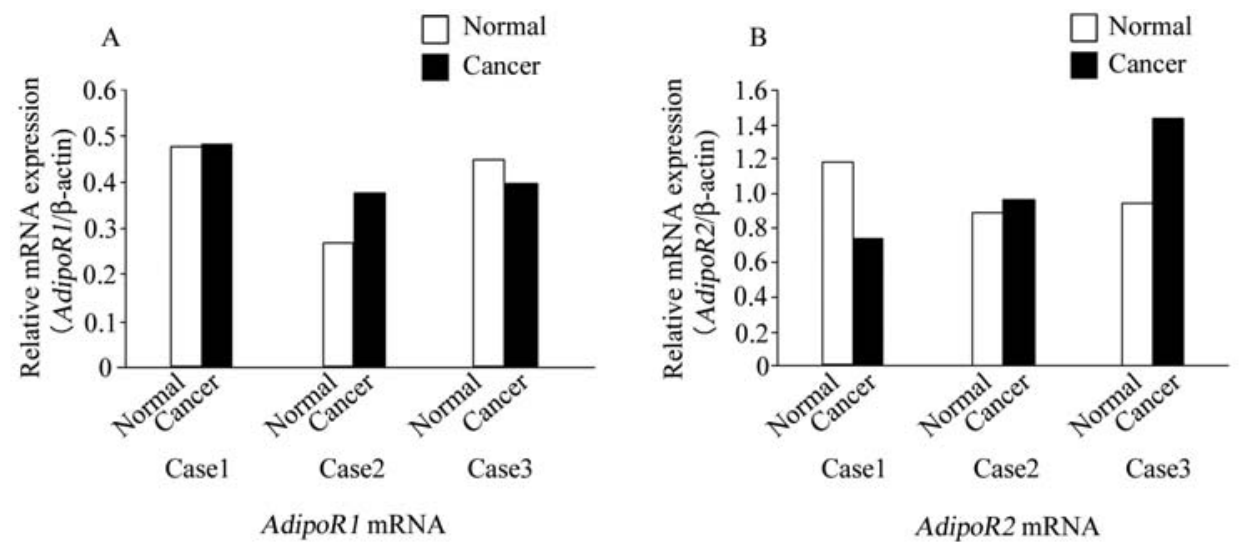

Figure 3. AdipoR1 (A) and AdipoR2 (B) mRNA expression levels of colonic epithelial cells and cancer cells collected from patients with cancer were examined with a laser microdissection system. Real-time RT-PCR was carried out. Values are normalized to the level of $\beta$-actin. All cell samples expressed AdipoR1 mRNA and AdipoR2 mRNA, the levels of expression of which were not significantly different between the normal cells and cancer cells.

Table I. Characteristics of cancer patients and control subjects.

\begin{tabular}{lccc}
\hline & Controls & Patients with cancer & P-value \\
\hline $\begin{array}{l}\text { Number } \\
\text { Location of }\end{array}$ & 18 & 16 & \\
cancer & & $\begin{array}{c}\mathrm{C}=1, \mathrm{~A}=2, \\
\mathrm{~T}=1, \mathrm{~S}=7, \mathrm{R}=5\end{array}$ \\
Age (years) & $59.89 \pm 2.4$ & $63.69 \pm 2.8$ & 0.3122 \\
$\begin{array}{l}\text { Gender } \\
\text { Male }\end{array}$ & 12 & 10 & \\
Female & 6 & 6 & \\
BMI $\left(\mathrm{kg} / \mathrm{m}^{2}\right)$ & $22.92 \pm 0.7$ & $22.44 \pm 1.0$ & 0.6934 \\
\hline
\end{tabular}

expressed the AdipoR1 and AdipoR2 proteins. The expression levels of the AdipoR1 (Fig. 1B) and AdipoR2 (Fig. 1C) proteins were not significantly different between the control and the cancer patients.

Gene expression analysis of AdipoR1 and AdipoR2. The expression levels of AdipoR1 and AdipoR2 mRNA were determined with a real-time RT-PCR assay (Fig. 2). All samples expressed AdipoRl and AdipoR2 mRNA. The levels of expression of AdipoR1 (Fig. 2A) and AdipoR2 (Fig. 2B) were not significantly different between the control and cancer specimens. In comparison with liver mRNA, the expression levels of normal colon epithelium AdipoRl and AdipoR2 were 33.5 and $22.8 \%$, respectively. The AdipoRl and AdipoR2 expression levels in CRC were 19.9 and $14.4 \%$, respectively (data not shown). In comparison with skeletal muscle mRNA, the expression levels of normal colon epithelium AdipoR1 and AdipoR2 were 13.1 and $13.5 \%$, respectively. Expression levels of CRC AdipoR1 and AdipoR2 were 7.8 and $7.9 \%$, respectively (data not shown). We also performed the full-length sequences of AdipoRs in cancer samples, though we could not find any mutation or deletion in these coding genes (data not shown). We investigated the relationship between plasma adiponectin concentration and the expression levels of the two AdipoRs, however we could not find any correlation between them (data not shown).

Laser microdissection and real-time RT-PCR of AdipoR1 and AdipoR2. Colonic epithelial cells and cancer cells were collected from three patients with cancer. All cell samples expressed AdipoR1 mRNA (Fig. 3A) and AdipoR2 mRNA (Fig. 3B). The expression levels of AdipoR1 and AdipoR2 


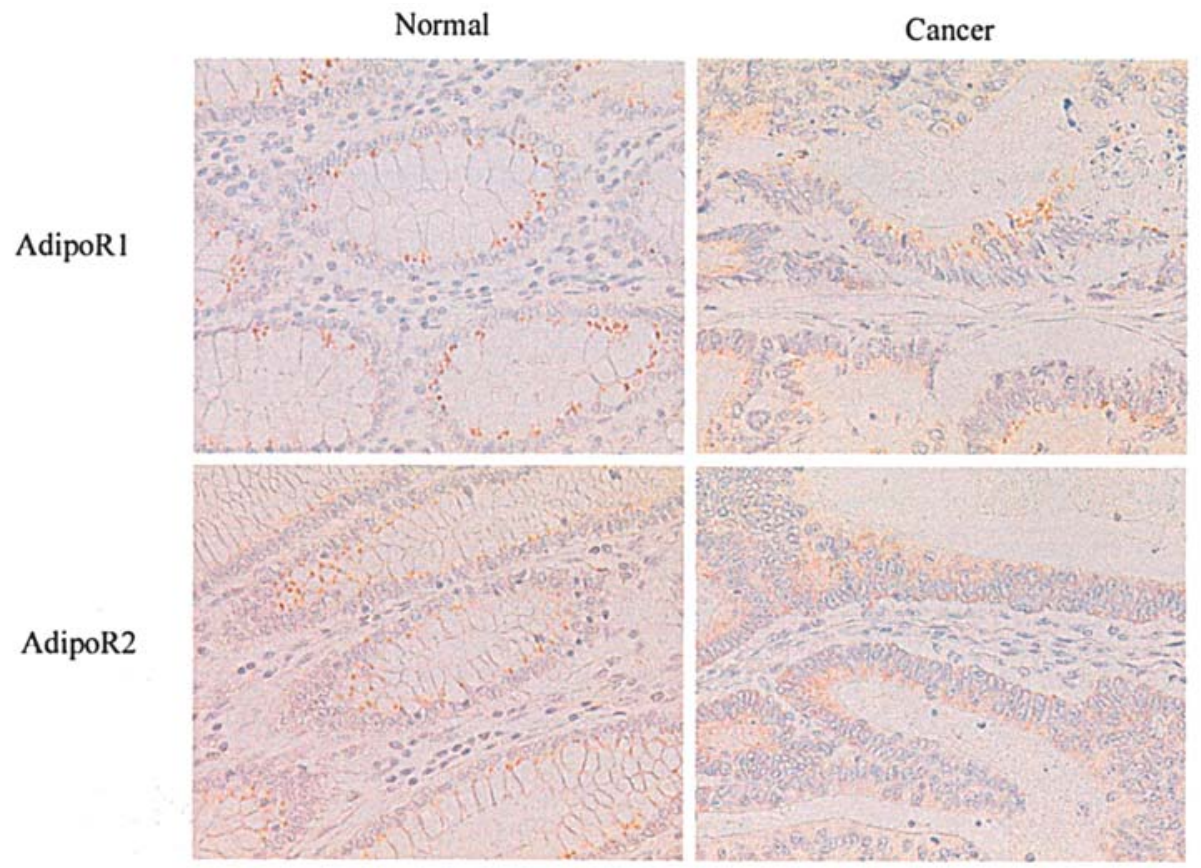

Figure 4. Immunohistochemical staining of AdipoR1 and AdipoR2 in normal and cancer tissue. AdipoR1 and AdipoR2 may be expressed in normal colonic epithelial cells and cancer cells. Concerning AdipoR1 and AdipoR2, strong staining of the cytoplasm can be observed in normal epithelial cells, primarily localized in the middle of the cells. Staining of a similar intensity is observed in cancer cells and normal cells.

mRNA were not significantly different between the normal cells and cancer cells.

Immunohistochemical staining of AdipoRl and AdipoR2. We evaluated the localization of the expression of the AdipoRs using immunohistochemical staining. AdipoRs were expressed in normal colonic epithelial cells and cancer cells (Fig. 4). Strong staining of the cytoplasm was observed in normal epithelial cells for AdipoR1 and AdipoR2, primarily localized in the vicinity of the nuclei. Staining of a similar intensity was observed in cancer cells and normal cells. In the cancer cells, diffuse cytoplasmic staining patterns were observed.

\section{Discussion}

In a prospective case-control study, Wei et al showed that men with lower plasma levels of adiponectin had a higher risk of CRC than those with higher plasma levels of adiponectin (12). In contrast, Lukanova et al reported finding no association between the plasma levels of adiponectin and the risk of CRC (13).

While some clinical studies regarding the plasma levels of adiponectin and $\mathrm{CRC}$ have been conducted on humans, no study has yet investigated the expression of the adiponectin receptors in normal colon epithelium and CRC tissues.

In the present human study, we clearly demonstrated the expression of adiponectin receptors, AdipoR1/AdipoR2, in normal colon epithelium and colon cancer cells. We confirmed our results at the genetic and protein expression levels and we further confirmed our results with laser microdissection. We could not find any mutation or deletion of the genes in the AdipoRs in this study. These results suggest the possibility that adiponectin may modulate the growth of normal colon epithelial and CRC cells directly through AdipoR1 or AdipoR2.

In order to identify the cell types which express the adiponectin receptors, we performed immunohistochemical staining using antibodies for the two AdipoRs and we showed the expression of these receptors in normal colon epithelial cells and CRC cells. These results being further confirmed by the laser microdissection analysis.

In regard to the relative expression ratios of AdipoR 1 and AdipoR2, the gene expression analysis showed they were equally expressed. Although the expression levels of AdipoR1 and AdipoR2 in the colon were lower in comparison to liver and skeletal muscle mRNA, it was sufficient to induce some effects.

In our study, we could not find any significant difference in the expression levels of the two AdipoRs between normal colon epithelium and CRC tissues. Notably, we were able to demonstrate the expression of AdipoRs even in advanced carcinoma. These results raise the possibility that adiponectin plays an important role in the promotion of colon cancer.

We showed that the two AdipoRs were expressed in advanced CRC tissues. These results suggest the possibility that the AdipoRs might be a novel therapeutic target for the treatment of CRC. Further study of the roles of adiponectin and the AdipoRs in normal colon epithelium and CRC should therefore be conducted and the downstream mechanism of the AdipoRs need to be elucidated.

In conclusion, we clearly demonstrated that the two adiponectin receptors, AdipoR1 and AdipoR2, were expressed in normal colon epithelium and CRC tissues using real-time RT-PCR analysis, Western blotting, laser microdissection analysis and immunohistochemical staining. To the best of our knowledge, this is the first study that demonstrates the 
expression of the adiponectin receptors in colon cancer tissue. Our results suggest the possibility that adiponectin might directly exert action on the promotion of colon cancer through the AdipoRs, which is different from the indirect action mediated by modulating the metabolic environment, such as improving insulin resistance. Further studies are required in order to elucidate the function of AdipoRs activated by adiponectin and the downstream mechanisms of the AdipoRs in colon cancer cells.

\section{Acknowledgements}

We thank Machiko Hiraga and Yuko Sato for their technical assistance. This study was supported in part by a Grant-in-Aid for research on the Third Term Comprehensive Control Research for Cancer from the Ministry of Health, Labour and Welfare, Japan to A.N., a grant from the National Institute of Biomedical Innovation (NBIO) to A.N., a grant from the Ministry of Education, Culture, Sports, Science and Technology, Japan (KIBAN-B) to A.N and a research grant of the Princess Takamatsu Cancer Research Fund to A.N.

\section{References}

1. Spranger J, Kroke A, Möhlig M, et al: Adiponectin and protection against type 2 DM. Lancet 361: 226-228, 2003.

2. Hotta K, Funahashi T, Arita Y, et al: Plasma concentrations of a novel, adipose-specific protein, adiponectin, in type 2 diabetic patients. Arterioscler Thromb Vasc Biol 20: 1595-1599, 2000.

3. Hu E, Liang P and Spiegelman BM: AdipoQ is a novel adipose-specific gene dysregulated in obesity. J Biol Chem 271: 10697-10703, 1996.

4. Gil-Campos M, Cañete RR and Gi A: Adiponectin, the missing link in insulin resistance and obesity. Clin Nutr 23: 963-974, 2004.
5. Maeda K, Okubo K, Shimomura I, Funahashi T, Matsuzawa Y and Matsubara K: cDNA cloning and expression of a novel adipose specific collagen-like factor, apM1 (AdiPose Most abundant Gene transcript 1). Biochem Biophys Res Commun 221: 286-289, 1996.

6. Yamauchi T, Kamon J, Waki H, et al: The fat-derived hormone adiponectin reverses insulin resistance associated with both lipoatrophy and obesity. Nat Med 7: 941-946, 2001.

7. Giovannucci E and Michaud D: The role of obesity and related metabolic disturbances in cancers of the colon, prostate, and pancreas. Gastroenterology 132: 2208-2225, 2007.

8. Dal Maso L, Augustin LS, Karalis A, et al: Circulating adiponectin and endometrial cancer risk: J Clin Endocrinol Metab 89: 1160-1163, 2004.

9. Miyoshi Y, Funahashi T, Kihara S, et al: Association of serum adiponectin levels with breast cancer risk. Clin Cancer Res 9: 5699-5704, 2003.

10. Mantzoros C, Petridou E, Dessypris N, et al: Adiponectin and breast cancer risk. J Clin Endocrinol Metab 89: 1102-1107, 2004.

11. Ishikawa M, Kitayama J, Kazama S, Hiramatsu T, Hatano K and Nagawa H: Plasma adiponectin and gastric cancer. Clin Cancer Res 11: 466-472, 2005.

12. Wei EK, Giovannucci E, Fuchs CS, Willett WC and Mantzoros CS: Low plasma adiponectin levels and risk of CRC in men: a prospective study. J Natl Cancer Inst 97: 1688-1694, 2005.

13. Lukanova A, Söderberg S, Kaaks R, Jellum E and Stattin P: Serum adiponectin is not associated with risk of CRC. Cancer Epidemiol Biomarkers Prev 15: 401-402, 2006.

14. Yamauchi T, Kamon J, Ito Y, et al: Cloning of adiponectin receptors that mediate antidiabetic metabolic effects. Nature 423: 762-769, 2003.

15. Takahata C, Miyoshi Y, Irahara N, Taguchi T, Tamaki Y and Noguchi S: Demonstration of adiponectin receptors 1 and 2 mRNA expression in human breast cancer cells. Cancer Lett 250: 229-236, 2007.

16. Michalakis K, Williams CJ, Mitsiades N, et al: Serum adiponectin concentrations and tissue expression of adiponectin receptors are reduced in patients with prostate cancer: a case control study. Cancer Epidemiol Biomarkers Prev 16: 308-313, 2007. 